\title{
The effects of figure/ground, perceived area, and target saliency on the luminosity threshold
}

\author{
FREDERICK BONATO \\ Saint Peter's College, Jersey City, New Jersey \\ and \\ JOSEPH CATALIOTTI \\ Ramapo College of New Jersey, Mahwah, New Jersey
}

\begin{abstract}
Observers adjusted the luminance of a target region until it began to appear self-luminous, or glowing. In Experiment 1, the target was either a face-shaped region (figure) or a non-face-shaped region (ground) of identical area that appeared to be the face's background. In Experiment 2, the target was a square or a trapezoid of identical area that appeared as a tilted rectangle. In Experiment 3 , the target was a square surrounded by square, circular, or diamond-shaped elements. Targets that (1) were perceived as figures, (2) were phenomenally small in area, or (3) did not group well with other elements in the array because of shape appeared self-luminous at significantly lower luminance levels. These results indicate that like lightness perception, the luminosity threshold is influenced by perceptual organization and is not based on low-level retinal processes alone.
\end{abstract}

Most surfaces appear opaque and illuminated by external light sources. However, some surfaces appear as light sources themselves. These surfaces are generally referred to as glowing, luminous or self-luminous (Bonato \& Gilchrist, 1994, 1999; Katz, 1935; Wallach, 1948). Although self-luminous surfaces are common in our visual experience, little is known about how the visual system detects them. Here we report results that deal specifically with the luminosity threshold - the luminance level at which opacity gives way to the appearance of self-luminosity.

The present study can be thought of as an extension of recent work conducted by Bonato and Gilchrist (1993, 1999) that showed how a target's perceived area affects the luminosity threshold. They measured luminosity thresholds for a small square target and a larger rectangular target. Both targets appeared to be on a large white background mounted onto a laboratory wall. The small target reached the luminosity threshold at a significantly lower luminance value than the large target, suggesting that as a target's area increases, that target's luminosity threshold also increases. In another experiment, they presented observers with one target or four targets embedded in an achromatic Mondrian display. The four targets' combined area was four times larger than the single target's area. Results showed that an increase in aggregate area can also raise the luminosity threshold.

In the two experiments described, target area varied both retinally and perceptually. In a third experiment, Bon-

Correspondence should be addressed to F. Bonato, Saint Peter's College, Department of Psychology, 2641 Kennedy Boulevard, Jersey City, NJ 07306 (e-mail: bonato_f@spcvxa.spc.edu). ato and Gilchrist (1999) measured luminosity thresholds for a target in a totally dark room under three viewing conditions: (1) a standard condition, (2) a condition in which the retinal area of the target was increased while its perceived area was kept the same, and (3) a condition in which the perceived area of the target was increased while its retinal area was kept the same. Results showed that increasing the perceptual area of the target significantly raised the target's luminosity threshold but increasing the target's retinal area did not.

One speculation that arose from this work concerns figure/ground relationships in the scene. It has been well established that small regions are more likely to appear as figures and larger regions are more likely to appear as ground (Goldhammer, 1934; Kunnapas, 1957; Oyama, 1950). In Experiment 1, we measured luminosity thresholds for two targets of equal retinal area; one of the targets was designed to appear more like a figure and the other was designed to appear more like ground.

\section{EXPERIMENT 1}

\section{Method}

Stimuli and Apparatus. The stimulus displays as seen by the observers are shown in Figure 1. The observer looked binocularly through a $2.5-\mathrm{cm} \times 13-\mathrm{cm}$ slot cut out from one end of an elongated box that was $38 \mathrm{~cm}$ wide, $31 \mathrm{~cm}$ high, and $74 \mathrm{~cm}$ long and saw a viewing chamber that was $38 \mathrm{~cm}$ wide, $31 \mathrm{~cm}$ high, and $41 \mathrm{~cm}$ deep, the interior of which was painted matte white. In the center of the viewing chamber's rear wall (reflectance $=90 \%, 6.7 \mathrm{~cd} / \mathrm{m}^{2}$ ) was a display that consisted of a face-shaped region and an adjacent region of equal area that appeared to be the face's background. On any given trial the brightness of either the face-shaped region or the non-face-shaped region was adjustable. Together these two regions formed a rectangle $\left(2.2 \mathrm{~cm} \times 2.9 \mathrm{~cm}, 2.8^{\circ} \times 3.6^{\circ}\right)$ that was in the center of a matte gray (reflectance $=16 \%, 1.7 \mathrm{~cd} / \mathrm{m}^{2}$ ) square 


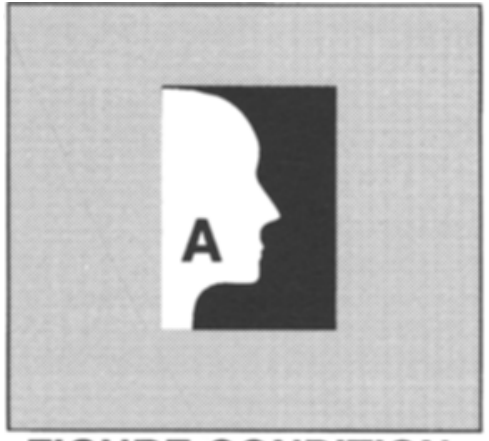

FIGURE CONDITION

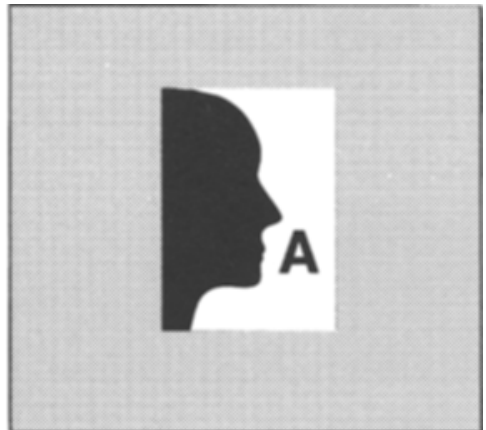

GROUND CONDITION

\section{$\mathbf{A}=$ adjustable brightness aperture}

Figure 1. The stimulus displays as seen by observers in Experiment 1.

$\left(11 \mathrm{~cm}^{2}, 13.5^{\circ}\right)$ of Color-Aid paper. When the face region's brightness was adjustable, the non-face-shaped region was a piece of matte black Color-Aid paper (reflectance $=3 \%, 0.5 \mathrm{~cd} / \mathrm{m}^{2}$ ). Conversely, when the non-face-shaped region's brightness was adjustable, the face region was a piece of matte black paper.

The apparatus is shown in Figure 2. Although the adjustable target region appeared to be located on the rear wall of the viewing chamber, the target was actually an aperture that opened up to a separately illuminated chamber positioned immediately behind the viewing chamber. The space that filled the aperture was a piece of matte gray Color-Aid paper (reflectance $=16 \%$ ) attached to the rear wall of the box. When looking through the viewing slot, the observer could pull two handles, one positioned on either side of the box, and increase the brightness of the aperture. If the observer pushed the two handles forward, the brightness of the aperture decreased. Each handle was attached to a sliding panel on each side of the apparatus. When pushed forward, the two panels moved in tandem and closed off two small chambers positioned on opposite side walls of the rear chamber. Each small chamber contained a 25 -cm-diameter 30-W circular fluorescent bulb. The small chambers' interiors were painted matte white to enhance light diffusion.

Illumination for the near viewing chamber was provided by two 4-W fluorescent bulbs mounted to the viewing chambers' interior of the viewing of chamber, one on each side of the viewing slot. The brightness of the bulbs was reduced and chromatic differences between surfaces in the viewing chamber and the aperture eliminated by covering the bulbs with acetate filters.

Procedure. The experimenter pushed the handles of the apparatus forward until the aperture appeared as a piece of black paper. The observer was led into the laboratory, seated in front of the apparatus, and given the following instructions:

In this experiment you are going to adjust the brightness of a region until it begins to appear self-luminous, or glowing. A glowing region is one that appears too bright to simply be a piece of white paper. Many times glowing regions appear to be emitting light. When I tell you to, I want you to look through the slot in front of you, grab hold of the two handles, one of which is on either side of the box, and slowly pull the handles toward you. When you pull the handles toward you, you will notice that the target region becomes brighter. At first it appears as a gray shade, but as you pull the handles toward you even further, it will appear white. If you continue to pull the handles toward you further, the target region will eventually begin to glow. Continuing to pull the handles will make the target region appear to glow even brighter.

Your task is to adjust the brightness of the target region until it just begins to appear luminous, or glowing. In other words, adjust the target until it appears too bright to be a piece of white paper. Make the variable region glow, but to the minimal possible degree. There is no time limit. Do you have any questions?

If the observer had no questions, he/she was instructed to look through the viewing slot and adjust the variable target region until it reached the luminosity threshold. When the observer was satisfied with his adjustment, he/she was then debriefed. When the observer had left the laboratory, the experimenter measured the luminance of the target aperture and changed the stimulus display as needed, before pushing the handles on the apparatus forward in preparation for the next observer.

Design. In the figure condition, 20 observers adjusted the luminance of the face-shaped aperture, and in the ground condition, 20 separate observers adjusted the luminance of the non-face-shaped aperture.

Observers. Forty experimentally naive undergraduate volunteers served as observers.

\section{Results and Discussion}

The individual luminosity thresholds obtained are shown in Figure 3. In the figure condition, the mean luminosity threshold for the face region was $29 \mathrm{~cd} / \mathrm{m}^{2}(\mathrm{~s}=$ $26, S E=5.9$ ). In the ground condition, the mean luminosity threshold for the non-face-shaped aperture was $67 \mathrm{~cd} / \mathrm{m}^{2}(s=78, S E=17.4)$. A $t$ test indicated that the mean threshold obtained in the figure condition (face) was significantly lower $[t(38)=2.26, p=.029]$ than the threshold obtained in the ground condition (non-faceshaped aperture). The physical and retinal areas of the targets in both conditions were identical. As can be seen in Figure 3 , one characteristic of these data is a high degree of between-subjects variability. This seems to be a general finding in luminosity threshold experiments. Other work using achromatic stimuli (Bonato \& Gilchrist, 1994, 1999) and chromatic stimuli (Evans, 1959; Speigle \& Brainard, 1996) has exhibited a high degree of variability.

Although quantitative size judgments for the two targets were not obtained, postexperimental reports unanimously indicated that (1) the face-shaped figural target appeared to be in front of the non-face-shaped region, and (2) the non-face-shaped ground target appeared larger than the face region because the non-face-shaped region appeared to extend behind the face region. These postexperimental 

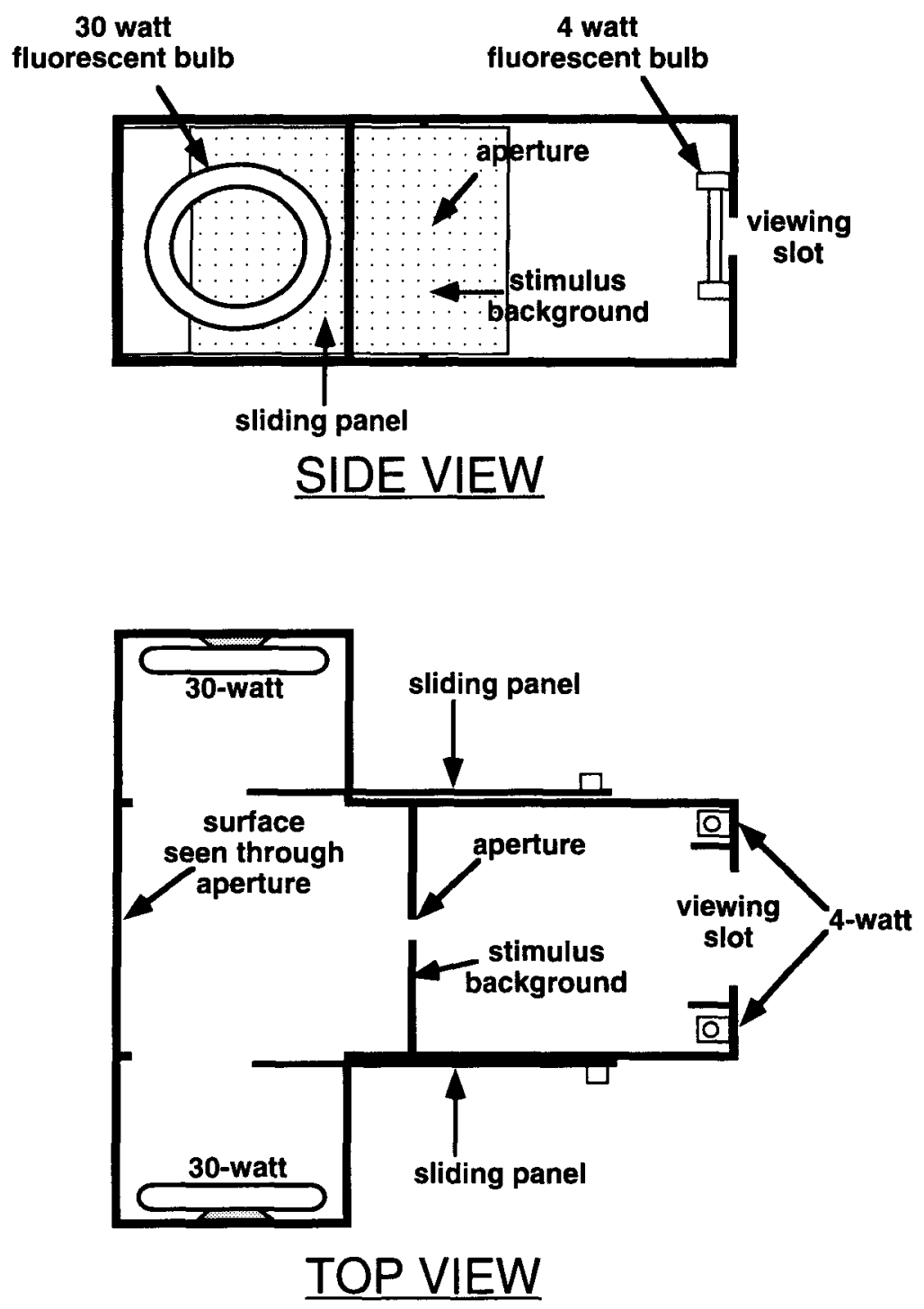

Figure 2. The apparatus used to obtain luminosity thresholds.

reports suggest that the face-shaped target appeared as figure and the non-face-shaped target appeared as ground.

It should be noted that Experiment 1 (and also 2 and 3) deals essentially with picture perception (Kennedy, 1974). All observers reported a dual experience when looking at our displays. They all agreed they were looking at a flat, two-dimensional display. However, they also agreed that in some sense, they had a three-dimensional experience when they looked at the displays, presumably because of the pictorial depth cues (linear perspective and texture gradient) that were present.

The results of Experiment 1 are not surprising when one considers two previous studies: (1) Coren's (1969) study, which showed that figural regions contrast more with their backgrounds than ground regions; and (2) Bonato and Gilchrist's (1994) study, which showed that the luminosity threshold in some ways behaves like a surface color and the uppermost boundary of the lightness dimension. Consider the two targets when their luminance level is equal to that of a surface perceived as white in the viewing chamber. The non-face-shaped (ground) target probably appears opaque white. The face-shaped (figure) target also appears white, but perhaps somewhat brighter than the ground target because of its increased level of perceived contrast (Coren, 1969). This superwhite appearance of the figure target, which Evans (1959) called fluorescence, and Heinemann (1955) called the enhancement effect, is a sort of intermediate between opaque white and the luminosity threshold. Because the figure target probably appeared brighter than the ground target, the figure target required less luminance to reach its luminosity threshold than the ground target. As the ground target's luminance increased, it had to first pass through the region of fluorescence before it reached the luminosity 


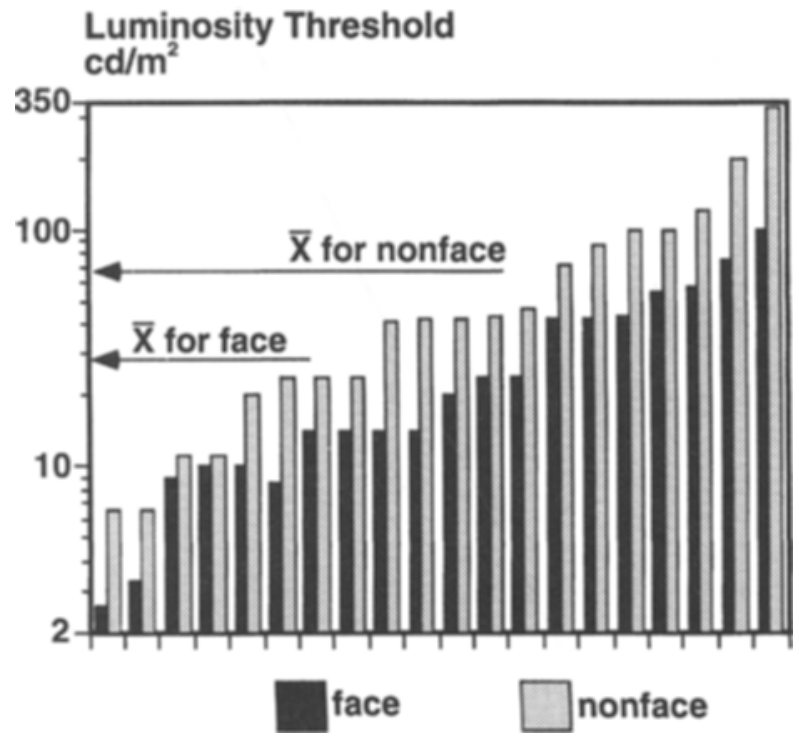

Figure 3. Individual luminosity thresholds obtained in Experiment 1.

threshold. However, the figure target already appeared brighter than the ground target; thus it required less luminance to appear self-luminous.

What is it about a figure region that tends to lower its luminosity threshold? Observers reported that the nonface-shaped region appeared larger than the face region because the non-face-shaped region appeared to extend behind the face region. This they reported because they perceived the figure (face) as being in front of the ground, which some described as a window behind the face. This description is in accordance with the phenomenal qualities of figure/ground outlined by Rubin (1921). Although we acknowledge that the perceived depth difference between the face-shaped and non-face-shaped regions may have been small, any difference in the perceived depth would be enough to perceive occlusion. This layered percept may have resulted in two target areas that appeared to have different areas even though the retinal areas of their corresponding images were identical. In Experiment 2, we focused our attention on perceived area independent of figure/ground differences.

\section{EXPERIMENT 2}

\section{Method}

Stimuli and Apparatus. The apparatus was identical to the one used in Experiment 1 except for the stimulus displays shown in Figure 4. One display consisted of a $1.9-\mathrm{cm}\left(2.4^{\circ}\right)$ square aperture and the other display consisted of a trapezoidal aperture $0.95-\mathrm{cm}$ wide at the top, $2.9-\mathrm{cm}$ wide at the bottom, and $1.9 \mathrm{~cm}$ high $\left(3.6^{\circ} \times 2.4^{\circ}\right)$. The areas of the two apertures were physically identical, and as a result also subtended the same amount of retinal area. Each aperture was centered within a $13-\mathrm{cm}$ square of matte gray (reflectance $=$ $16 \%, 1.7 \mathrm{~cd} / \mathrm{m}^{2}$ ) Color-Aid paper that contained a pattern of black horizontal lines (reflectance $=3 \%, 0.5 \mathrm{~cd} / \mathrm{m}^{2}$ ) and that was mounted in the center of a matte white (reflectance $=90 \%$, $6.7 \mathrm{~cd} / \mathrm{m}^{2}$ ) background panel. The black horizontal lines decreased in width toward the top of the display, creating a texture gradient that together with the linear perspective of the trapezoidal aperture produced the impression that the aperture was not a trapezoid, but a rectangle whose top was tilted backward and away from the observer. This display was created to alter the perceived size of the target aperture while keeping the visual areas of the two targets the same

Two cardboard Ls were used to obtain size judgments for the aperture targets. The two Ls could be held together to form a rectangular window whose size could be adjusted by sliding the two Ls against each other.

Procedure. The procedure was identical to that used in Experiment 1 with the following exceptions: (1) If the observer adjusted the brightness of the trapezoidal target, he/she was asked afterward if the target appeared as trapezoid or a tilted rectangle; and (2) size judgments were obtained for the target using the two cardboard Ls The observer was instructed to imagine taking the target out of the chamber and placing it against the cardboard Ls. He/she was then asked to make the opening formed by the two Ls the same size as the target. The observer was allowed to look back and forth between the target and the opening formed by the cardboard Ls, and (3) when the observer was satisfied with his/her size judgment, the experimenter measured and recorded the dimensions of the window the observer created with the two Ls.

Design. One group of 20 observers adjusted the luminance and made size judgments for the square target aperture, and a separate group of 20 observers did the same for the trapezoidal target aperture.

Observers. Forty experimentally naive undergraduate volunteers served as observers.

\section{Results and Discussion}

The individual thresholds obtained are shown in Figure 5. The mean luminosity threshold for the square aperture was reached at $41 \mathrm{~cd} / \mathrm{m}^{2}(s=30, S E=6.8)$. The mean luminosity threshold for the trapezoidal target was reached at $144 \mathrm{~cd} / \mathrm{m}^{2}(s=111, S E=25)$. A $t$ test indicated that the mean threshold obtained in the trapezoid condition was significantly higher $[t(38)=3.36, p=.002]$ than the threshold obtained in the figure condition. The mean size judgment for the trapezoidal target aperture was $17.7 \mathrm{~cm}^{2}(s=15, S E=3.2)$, which was also significantly higher $[t(38)=3.9, p=.000]$ than the mean size judgment of the square target aperture, which was $4.9 \mathrm{~cm}^{2}(s=1.3$, $S E=0.3$ ).

As noted, the two target apertures produced retinal images of equal area, but as the size judgment results suggest, the trapezoidal target aperture could be seen in such a way (as a rectangle) that its perceived area was greater than that of the square target aperture. As stated in the discussion of Experiment 1, these experiments deal essentially with picture perception and the dual experience that many pictures elicit. All observers reported that they could perceptually organize the trapezoidal target in such a way so that it appeared as a rectangle, but when asked if the target was really a rectangle, they all agreed that it was not. These results are in agreement with those obtained by Bonato and Gilchrist (1999); however, their subjects had other depth cues available to them (stereopsis, accommodation, and convergence), whereas depth information in our displays was provided entirely by pictorial cues - namely, linear perspective and a texture gradient.

Another notable difference between the results of Experiment 2 and the results obtained by Bonato and Gil- 


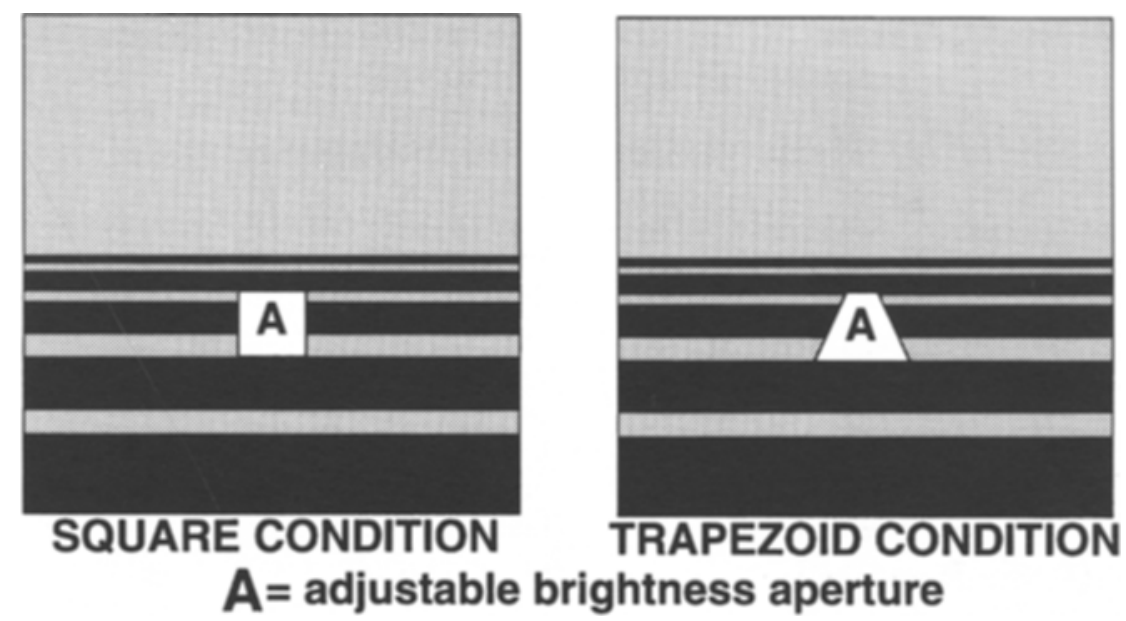

Figure 4. The stimulus displays as seen by observers in Experiment 2.

christ (1999) is the apparent size of the effect. The area of the trapezoidal target in Experiment 2 was judged by observers to be about 3.5 times larger than the area of the square target. The mean luminosity threshold obtained for the trapezoidal target was also about 3.5 times higher than the mean threshold obtained for the square target. In Bonato and Gilchrist's experiment, the area of the perceptually larger target was judged by observers to be about 12 times larger than the area of the standard target. However, the mean luminosity threshold obtained for the perceptually larger target was only about 2 times higher than the mean threshold obtained for the standard target. Although we cannot say for sure why there was a difference in magnitude of the area effects obtained in the two experiments, we can say that the conditions of the two experiments were very different, allowing perhaps for other variables to exert an influence on the luminosity thresholds. For example, the stimulus displays that were presented in Bonato and Gilchrist's experiment consisted of simple target/background displays in a dark void. Under these conditions, one could argue that the whole display appeared self-luminous.

There is a possible alternative to a perceived area explanation for the results of Experiment 2-namely, that the difference in perceived orientation of the targets was responsible for the differences in the obtained thresholds. Perceived orientation might make a difference because it has been claimed (Ramachandran, 1988; Ramachandran \& Kleffner, 1992) that there is a tendency for humans to perceive the illuminant as coming from above. In a lightness perception experiment, Hochberg and Beck (1954) placed a vertical trapezoidal target on a table directly below an overhead light and found that when the target was seen as a rectangle lying flat on the table, it was judged as darker than when the target was seen as a vertical trapezoid. Later replications (Beck, 1965; Flock \& Freedberg, 1970) measured the effect and found it to be weak, about one half a Munsell step. One attempt at repli- cation (Epstein, 1961) revealed no effect of the target's perceived orientation.

In order to rule out the possibility that the results of Experiment 2 were due to a combination of perceived orientation of the targets and a tendency to perceive the illuminant as coming from above, we showed some observers the exact same displays used in Experiment 2, only the displays were presented upside down. This resulted in another problem, however: Some observers who viewed the upside down displays reported that the trapezoidal target appeared as ceiling light fixture, a situation that would tend to lower its luminosity threshold in a way that we had not anticipated. Observers who viewed the displays sideways found the displays too difficult to organize.

Recently we (Policastro, Bonato, \& Cataliotti, 1998) conducted a lightness perception experiment using the

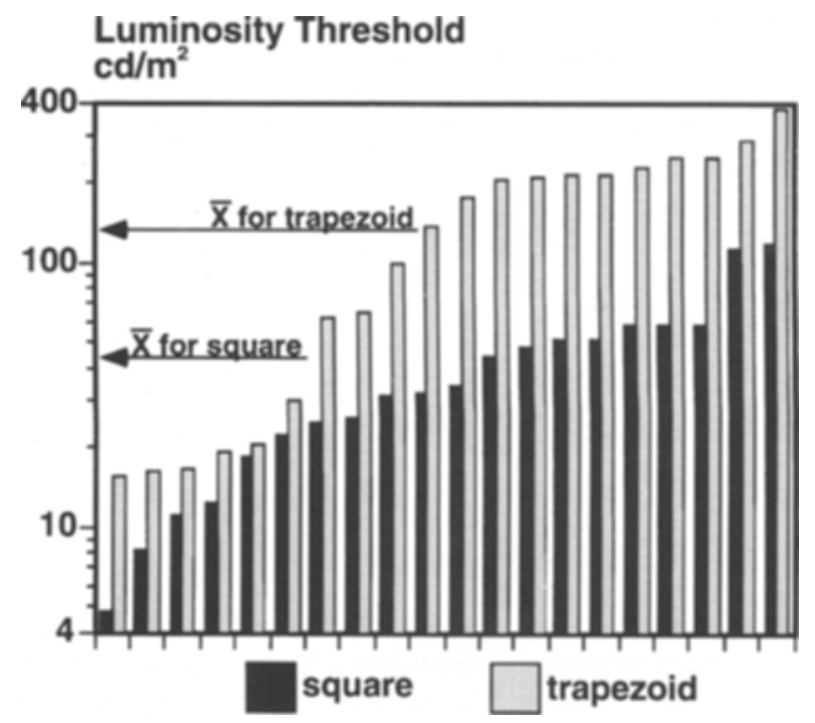

Figure 5. Individual luminosity thresholds obtained in Experiment 2. 

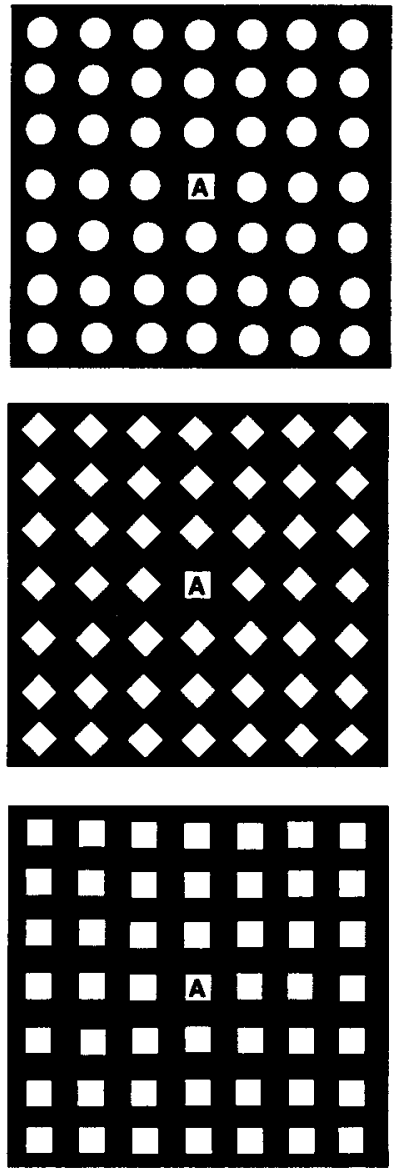

Figure 6. The stimulus displays as seen by observers in Experiment 3.

same displays used in the present Experiment 2, the results of which do not support the idea that perceived orientation of the targets plays a role. Both the square target and the trapezoidal target were set to a luminance value well within the opaque gray range. Observers matched the target's lightness with a chart of 16 Munsell chips that ranged from black (reflectance $=3 \%$ ) to white (reflectance $=90 \%$ ). The mean lightness match for the square target $(7 \%)$ was not significantly different $[t(38)=$ $0.69, p=.49]$ from the mean match obtained for the trapezoidal target (7.8\%). Postexperimental inquiries indicated that observers viewing our displays did not consciously perceive the illuminant as coming from above. If the illuminant was perceived as coming from above in Experiment 2, and this was the variable responsible for the higher luminosity threshold obtained in the trapezoid condition, the trapezoidal target in our lightness experiment should have been matched to a darker shade of gray on the Munsell chart, but this did not happen. These results do not contradict Bonato and Gilchrist's (1994) results, which showed that the luminosity threshold, like opaque surfaces, exhibits constancy relative to changes in the illumination. However, unlike the luminosity threshold, the opaque targets were unaffected by perceived size, demonstrating that the luminosity threshold does not behave like a surface color in all ways.

These results put into question whether figure/ground relationships per se can affect the luminosity threshold. Bonato and Gilchrist (1999) attempted to make a target more groundlike by placing a decrement (darker than its surround) in the target's center. Contrary to the results of the present Experiment 1, their target, which was the background to a square, had a lower luminosity threshold than a homogenous target. But if the real effect of figure/ground on the luminosity threshold is based on perceived size, their results make sense. When they placed a small square on their large rectangular target, they may very well have made the target more groundlike, but the perceived area of the target was probably less than the perceived area of the homogeneous target. Although the target's surface appeared to extend behind the square, this perceptual extension was probably not complete. Kanizsa (1979) has shown that there is a perceptual shrinkage of an occluded region, and Shimojo and Nakayama (1990) have demonstrated analogous results using an apparent motion display.

In Experiment 3, we tested the effect of target saliency on the luminosity threshold. It is well known by now, due mainly to the work of Treisman and her colleagues (Treisman, 1988; Treisman \& Gelade, 1980), that some targets perceptually "pop out" from an array because of a distinctive feature. "Pop-out" is not meant to imply a segregation in depth. A pop-out target is one that requires less time to locate in a visual search. In Experiment 3, we tested the effect of perceptual pop-out on the luminosity threshold.

\section{EXPERIMENT 3}

\section{Method}

Stimuli and Apparatus. The apparatus was the same as in the previous experiments with the exception of the stimulus displays. The three displays used are shown in Figure 6. Each display consisted of a $25-\mathrm{cm}$ square of matte black (reflectance $=3 \%$, $0.5 \mathrm{~cd} / \mathrm{m}^{2}$ ) Color-Aid paper mounted onto the center of the white $\left(6.7-\mathrm{cd} / \mathrm{m}^{2}\right)$ rear wall of the viewing chamber. Three sets of 48 array elements made of white (reflectance $=90 \%, 6.7-\mathrm{cd} / \mathrm{m}^{2}$ ) Color-Aid paper, all of which had the same area $\left(3.6 \mathrm{~cm}^{2}\right)$, were arranged on the black square in rows and columns: $2.1-\mathrm{cm}$-diameter circles, 1.9 $\mathrm{cm}$ diamonds, or 1.9-cm squares. In the center of each display was a $1.9-\mathrm{cm}^{2}$ adjustable brightness aperture.

Procedure. The procedure was the same as that used in Experiment 2, with the following two exceptions: (1) size judgments for the target were not recorded, and (2) during debriefing, observers were shown the three displays and asked which aperture appeared to be more like a figure.

Design. Twenty observers set luminosity thresholds for the aperture surrounded by circular elements: a second group of 20 did the same for the aperture surrounded by diamond shaped elements, and a third separate group of 20 did the same for the aperture surrounded by square array elements.

Observers. Sixty experimentally naive undergraduate volunteers served as observers.

\section{Results and Discussion}

The individual thresholds obtained in Experiment 3 are shown in Figure 7. The mean luminosity thresholds obtained for the target apertures surrounded by circular, 


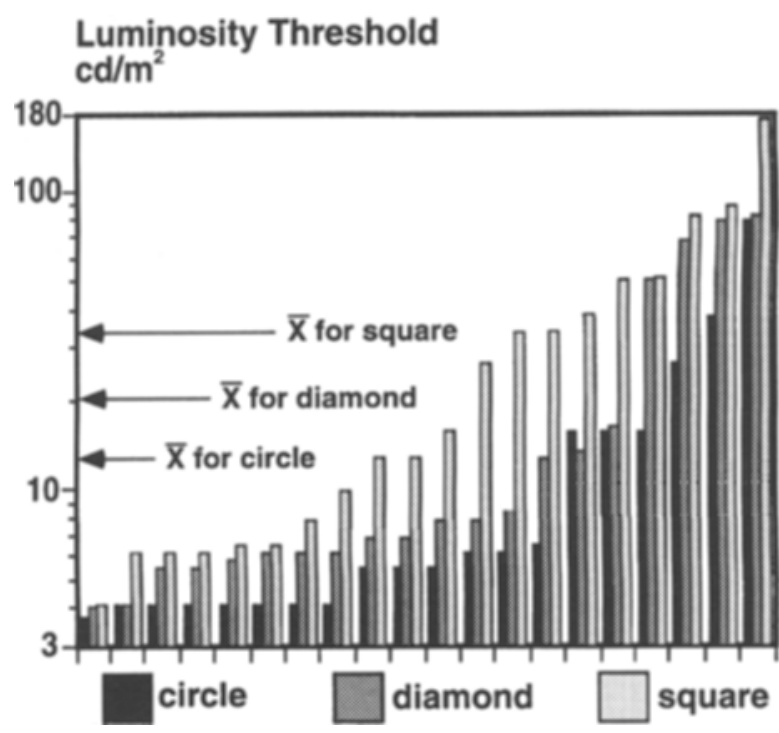

Figure 7. Individual luminosity thresholds obtained in Experiment 3.

diamond, and square array elements were $12.9(s=18$, $S E=4.0), 20.2(s=26, S E=5.9)$, and $33.4 \mathrm{~cd} / \mathrm{m}^{2}(s=41$, $S E=9.2$ ), respectively. A one-way analysis of variance indicated a significant difference between these mean thresholds $[F(2,57)=3.79, p=.028]$, and a Tukey's HSD post hoc test indicated that the difference lies between the thresholds obtained in the circular array elements condi- tion and the square array elements condition. These results suggest that a pop-out region that does not group well with its background will have a lower luminosity threshold than a region that blends in, even if the two regions are perceived to have the same area.

Is it fair to say that a target that is more perceptually salient appears more like a figure than a target that groups well with other elements in an array because of similarity? And, have we found a case where figure/ground relations affect the luminosity threshold independently of perceived size? During the debriefing process, observers were shown all three stimulus displays and asked which aperture appeared to be the most like a figure. They unanimously agreed that the square target aperture surrounded by circles appeared to be the most figurelike target because it stood out from the array. However, even though most of the observers were psychology students, we do not know how familiar they were with the characteristics of figures outlined by Rubin (1921).

One characteristic of figures outlined by Rubin (1921) is that the border that separates figure from ground tends to perceptually belong to the figure. It should be noted, however, that Rubin stated that this was not always the case. Recent investigators (Baylis \& Driver, 1995) have also supported the notion that the contour that separates figure and ground tends to be perceptually grouped with the figure. We believe that in all three conditions of Experiment 3 , the border that separated each target aperture from its background appeared to belong to the aperture. Although we did not test this, it appears self-evident when one looks at the displays, and if true, it means that in

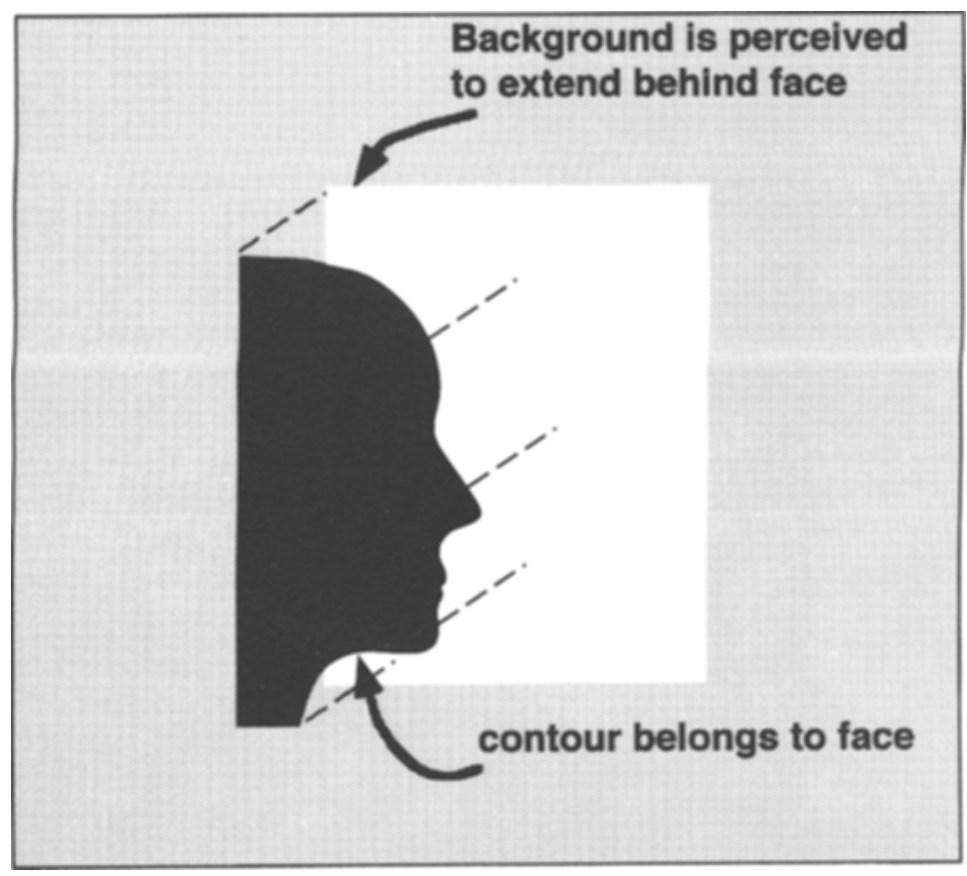

Figure 8. The layered percept of a figure/ground display. 
terms of this particular characteristic, all three apertures appeared as figures. What does appear clear from the results of Experiment 3 is that increasing target saliency via perceptual grouping can influence the luminosity threshold of a target region.

\section{GENERAL DISCUSSION}

In these experiments, we have shown that surfaces perceived as figures will reach the luminosity threshold at a lower luminance level than surfaces of equal physical area that are perceived as ground. These results are in agreement with Coren's (1969) work, in which he found a greater contrast effect for surfaces perceived as figures. Recent work by Gerbino and Nicolosi (1996) showed that the convexity of a target can also play a role when a subject searches for a target embedded in an array of distractor elements. In a visual search task, they found that convex targets were detected at a higher rate than concave targets. Their interpretation is that convexity increased the figurelike quality of the targets. This manipulation in turn made the concave targets appear brighter than the concave targets, perhaps even self-luminous.

However, figure/ground relations are frequently coupled with changes in perceived size due to the layered percept that is created whenever a figure is seen against ground. In general, the figure is perceived to be in front of the ground, and as a result, the ground is perceived to extend behind the figure. This layered type of percept, which was reported by all the observers in Experiment 1 and is depicted in Figure 8, suggests that differences in the thresholds obtained for the figure targets and ground targets presented in Experiment 1 may have been at least partially caused by differences in the perceived areas of the two targets. This hypothesis is in agreement with results obtained by Bonato and Gilchrist (1999) showing that the perceived area of a region is important in determining its luminosity threshold. Comparing target regions having equal retinal areas but different perceived areas, they found that as the perceived area of a surface increased, that surface's luminosity threshold also increased. However, they found no effect when retinal area was changed independently.

The notion that perceived size is important is supported by the results of Experiment 2, in which figure/ground relationships were held constant, but perceived size was varied. Unlike the observers who served in Bonato and Gilchrist's (1999) experiment, who had accommodation, vergence, and stereoscopic information available to them, the observers who served in Experiment 2 had only pictorial information available to them, which included linear perspective and texture gradients. The results of Experiment 2 show that pictorial cues alone can affect the luminosity threshold.

The results of Experiment 3 indicate that pop-out targets that are perceptually more salient than other elements in an array have a lower luminosity threshold. It is debatable whether making a target more salient also makes it more figural. However, the results of Experiment 3 indi- cate that other grouping principles can play a role in determining a surface's luminosity threshold, in this particular case, grouping by similarity.

Overall, these results show that perceptual organization and perceived characteristics of the stimulus play an important role in luminosity perception, and the luminosity threshold is not based on photometric information alone. As is the case in lightness perception, scene geometry and perceptual grouping also play a role (Gilchrist \& Bonato, 1995; Gilchrist et al., 1999; Li \& Gilchrist, 1999). This is not surprising because it has been shown (Bonato \& Gilchrist, 1994, 1999) that in some ways the luminosity threshold behaves like a surface color itself, occupying the very top of the black-to-white lightness scale.

Although these results show how figure/ground relations, perceived size, and perceptual grouping can influence the luminosity threshold, they do not show why these factors influence luminosity perception. Because surfaces perceived as self-luminous in our everyday experience are frequently light sources, we can only say that our results somewhat mirror that which exists in the real world. Specifically, light sources are usually objects (figures) and not backgrounds, and light sources are usually small relative to their surrounds. We cannot speculate at this time whether the tendencies we show in this paper are the product of genetic hardwiring or some sort of perceptual learning.

The results reported in this study are psychophysical in nature. However, when psychoanatomical reasoning is applied, these results provide clues as to where the processing required for luminosity perception takes place. Because these experiments show that perceived characteristics of the visual field are important in determining the luminosity threshold, explaining luminosity perception in physiological terms will be a difficult task. In light of the present findings, it appears that the search for underlying physiological explanations should not be confined to the retina. Instead, the search should be extended higher up the visual pathway. Given that the luminosity threshold seems to be influenced by several factors, a complete physiological explanation will probably include several areas of the brain. Perhaps a more difficult task will be describing how these different areas interact with one another and operate as an integrated whole.

\section{REFERENCES}

Baylis, G., \& Driver, J. (1995). One-sided edge assignment in vision: 1. Figure-ground segmentation and attention to objects. Current Directions in Psychological Science, 4, 140-146.

BECK, J. (1965). Apparent spatial position and the perception of lightness. Journal of Experimental Psychology, 69, 170-179.

Bonato, F., \& Gilchrist, A. (1993). The effect of target size and number on the perception of luminosity [Abstract]. Investigative Ophthalmology \& Visual Science, 34, 781.

Bonato, F, \& Gilchrist, A. L. (1994). The perception of luminosity on different backgrounds and in different illuminations. Perception, 23, $991-1006$.

Bonato, F., \& Gilchrist, A. L. (1999). Perceived area and the luminosity threshold. Perception \& Psychophysics, 61, 786-797.

COREN, S. (1969). Brightness contrast as a function of figure-ground relations. Journal of Experimental Psychology, 80, 517-524. 
EPSTEin, W. (1961). Phenomenal orientation and perceived achromatic color. Journal of Psychology, 52, 51-53.

EvaNs, R. M. (1959). Fluorescence and gray content of surface colors. Journal of the Optical Society of America, 54, 1049-1059.

Flock, H. R., \& Freedberg, E. (1970). Perceived angle of incidence and achromatic surface color. Perception \& Psychophysics, 8, 251256.

Gerbino, W., \& Nicolosi, L. (1996). Meaning affects preattentive figure/ground organization [Abstract]. Investigative Ophthalmology \& Visual Science, 37, S14.

Gilchrist, A., \& Bonato, F. (1995). Anchoring of lightness values in center/surround displays. Journal of Experimental Psychology: Human Perception \& Performance, 21, 1427-1440.

Gilchrist, A., Kossyfidis, C., Bonato, F., Agostini, T., Cataliotti, J., Li, X., Spehar, B., Annan, V., \& Economou. E. (1999). An anchoring theory of lightness perception. Psychological Review, 106, 795-834.

GOLDHAMmer, H. (1934). The influence of area, position, and brightness in the perception of a reversible configuration. American Journal of Psychology, 46, 189-206.

HeinemanN, E. G. (1955). Simultaneous brightness induction as a function of inducing- and test-field luminances. Journal of Experimental Psychology, 50, 89-96.

HoCHBERG, J. E., \& BECK, J. (1954). Apparent spatial arrangement and perceived brightness. Journal of Experimental Psychology, 47, 263266.

Kanizsa, G. (1979). Organization in vision. New York: Praeger.

KATZ, D. (1935). The world of colour. London: Kegan Paul.

KENNEDY, J. M. (1974). A psychology of picture perception, images, and information. San Francisco: Jossey-Bass.

KUNNAPAS. T. (1957). Experiments on figural dominance. Journal of Experimental Psychologv, 53, 31-39.
LI, X., \& Gilchrist, A. L. (1999). Relative area and relative luminance combine to anchor surface lightness values. Perception \& Psychophysics, 61, 771-785.

OYAMA, T. (1950). Figure-ground dominance as a function of sector angle, brightness, hue, and orientation. Journal of Experimental Psychology, 60, 299-305.

Policastro, L., Bonato, F., \& Cataliotti, J. (1998). Does the luminosity threshold depend on perceived area or the perceived direction of the illuminant? Paper presented at the annual meeting of the Eastern Psychological Association, Boston.

RamaChandran, V. S. (1988). Perception of shape from shading. $\mathrm{Na}$ ture, 331, 163-166.

RamaChandran, V. S., \& KlefFner, D. A. (1992). On the perception of shape from shading. Perception \& Psychophysics, 52, 18-36.

RUBIN, E. (1921). Visuell wahrgenomenne Figuren [Visually perceived figures]. Copenhagen: Gyldendal.

Shimojo, S., \& Nakayama, K. (1990). Amodal presence of partially occluded surfaces: Role of invisible stimuli in apparent motion correspondence. Perception, 19, 285-299.

Speigle, J. M., \& Brainard, D. H. (1996). Luminosity thresholds: Effects of test chromaticity and ambient illumination. Journal of the Optical Society of America A, 13, 436-451.

Treisman, A. M. (1988). Features and objects: The Fourteenth Bartlett Memorial Lecture. Quarterly Journal of Experimental Psychology, 40A, 201-237.

Treisman, A. M., \& Gelade, G. (1980). A feature-integration theory of attention. Cognitive Psychology, 12, 97-136.

WALLACH, H. (1948). Brightness constancy and the nature of achromatic colors. Journal of Experimental Psychology, 38, 310-324.

(Manuscript received April 4, 1997; revision accepted for publication November $25,1998$. 